\title{
Buchrezension: Roald Amundsen's Sled Dogs: The Sledge Dogs Who Helped Discover the South Pole
}

\author{
Cornelia Lüdecke \\ Universität Hamburg, Hamburg, Deutschland \\ Correspondence: Cornelia Lüdecke (c.luedecke@lrz.uni-muenchen.de)
}

Published: 21 May 2021

Tahan, M. R.: Roald Amundsen's Sled Dogs: The Sledge Dogs Who Helped Discover the South Pole, Springer Nature Switzerland AG, Cham, 640 pp., ISBN 978-3-030-02691-2, EUR 51.99, 2019.

Um es gleich am Anfang zu sagen: Wer dieses Buch liest, muss starke Nerven haben, denn es geht um den unbarmherzigen Ge-und Verbrauch von grönländischen Schlittenhunden, die Roald Amundsen während des legendären Wettlaufs zum Südpol (1911-1912) verwendet hat. Man muss es mögen, immer wieder von taktischen Tötungen der Hunde zu lesen, deren Kadaver wiederum den anderen Hunden zu Fraß vorgeworfen wurden oder auch der Sättigung des menschlichen Hungers dienten. Um was geht es der kanadischen Autorin Mary Tahan eigentlich dabei? Sie möchte Amundsens Hunden, ohne die er es nie als erster zum geographischen Südpol geschafft hätte, ein Denkmal setzen. In dem vorliegenden Buch hat sie offenbar wirklich alles Wissenswerte über die Hunde zusammengetragen. Dafür wertete sie alle verfügbaren Tagebücher der Teilnehmer an Amundsens Südpolexpedition und zusätzliche Korrespondenzen sowie Amundsens Filmaufnahmen aus und stellte sie Amundsens publiziertem offiziellen Reisebericht gegenüber, der manche Episoden im Gegensatz zu den Beobachtungen der anderen Expeditionsteilnehmer verschweigt, verharmlost oder geschönt widergibt.

Damit die Kapitel auch einzeln elektronisch vom Verlag erworben werden können, beginnen sie mit einer Zusammenfassung und enden mit den Hinweisen auf die benutzen Quellen sowie meistens auch mit einer jeweils aktualisierten Liste der lebenden Hunde, ihrem Nachwuchs, ihrer Einteilung in die einzelnen Schlittenteams, ihrem tatsächlichen Einsatz für die Schlittenreisen und wann sie starben, bzw. getötet wurden. Bilder der Hunde und Fotos von Schriftstücken, die aber nicht immer lesbar sind, runden die einzelnen Kapitel ab. Leider gibt es keinen Orts- und Namensindex, der einem helfen könnte, einzelne Stellen in dem umfangreichen Buch wiederzufinden. Eine Karte mit den Angaben der einzelnen Depots zwischen der Überwinterungsstation Framheim und dem Südpol wäre zudem eine recht nützliche Beigabe gewesen.

Die Autorin beschreibt zunächst Amundsens erste Erfahrungen in den Polargebieten als Mitglied der belgischen Antarktisexpedition (1897-1899), während seiner Befahrung der Nordwestpassage (1903-1906) und wie er nach der Erreichung des geographischen Nordpols durch Frederick Cook und Robert Peary spontan umdisponierte und sich nun anstelle des Nordpols den Südpol zum Ziel nahm. Während Scott vorhatte, neben Hunden und Ponys auch Motorschlitten zu verwenden, entschied sich Amundsen zum Kauf der besten Schlittenhunde der Welt, die er von der Grönländischen Handelskompanie bezog.

Die erste Prüfung für die Hunde besteht aus der rauen Überfahrt von Grönland nach Norwegen, der die lange Reise über den Äquator zur Antarktis folgt. Es wird beschrieben, wie sich die Mannschaft unterwegs rührend um die Hunde und ihren Nachwuchs kümmert, denn von ihnen würde ja der Erfolg der Expedition abhängen. Alle potentiellen Hundeschlittenführer haben an Bord die Aufgabe, ihren Hunden alle Fürsorge angedeihen zu lassen. An dieser Stelle des Buches wird auch die erste Liste von neun Hundeteams mit allen von der Autorin recherchierten Hundenamen aufgeführt. Bald kommt es wegen läufiger Hündinnen zu Problemen an Bord. Bereits hier beginnt Amundsen, nüchternen Überlegungen folgend, gezielt weibliche Welpen zu töten, damit nur sinnvoll nutzbare Tiere, d.h. männliche Welpen, als Schlittenhunde großgezogen werden. In der ersten Hälfte des Bu- 
ches wird die emotionale Bindung der Expeditionsteilnehmer zu ihren Hunden groß geschrieben.

In der zweiten Hälfte, als die Schlittenreise in Richtung Südpol viel zu früh schon bei der Wiederkehr des Sonnenlichts startet und aufgrund erneuter sehr tiefer Temperaturen umkehren muss, kippt die Stimmung in Framheim. Nun muss man von der engen Beziehung des Menschen zum Hund absehen und völlig emotionslos Amundsens Handeln betrachten, der nur sein Ziel im Auge hat. Er selbst ist ein miserabler Hundeschlittenführer, der durch den verfrühten Aufbruch in der großen Kälte sein eigenes Hundeteam an Überanstrengung verloren hat. Er sieht die Hunde jetzt nicht mehr als Individuen mit emotionalem Wert sondern als Bestandteil einer Möglichkeit, möglichst schnell auf Schnee und Eis von A nach B vorzudringen. Wenn die Hunde dabei nicht mehr funktionieren und dann nutzlos sind, werden sie einfach getötet und als Nahrung für Tier und Mensch weiterverwendet.

Zudem hatte Amundsen genau berechnet, wie viel Hundefutter er maximal mitnehmen muss, damit die Hunde auch bei halben Futterportionen ausreichend weit vorwärts kommen, bevor die Hundezahl beim Erreichen des antarktischen Plateaus am Rastplatz „Butcher’s Shop“ (,Metzgerei“) - allein schon der Name dieses Haltepunktes spricht Bände auf ein Minimum reduziert wird. Alle Schlittenführer außer Amundsen selbst müssen sich an dem Massaker teilnehmen. Unter großer Anstrengung, mit wenig Futter aber mit mehr Schlägen wird der Südpol mit 17 Hunden erreicht, von denen jedoch ein schwaches Tier am Ziel getötet wird.

Neben der Beschreibung der Hundeschicksale wird durch den Vergleich der einzelnen Tagebucheinträge der Teilnehmer auch ein detailliertes Bild von Amundsens spontanen Entscheidungen als Reaktion auf unvorhergesehene Ereignisse gegeben. So erfährt man mehr über Hjalmar Johansens Aufbegehren gegen Amundsen nach seinem verheerenden führen Aufbruch zum Südpol, die anschließende einmonatige Erholungsphase und erneute Vorbereitung für die Südpolreise, den dreitägigen Aufenthalt am Südpol, die Suche nach dem rettenden Depot auf der Heimreise beim Abstieg vom Eisplateau, Amundsens ständig aktualisierte Notfallpläne und vieles mehr.
Schließlich werden die Überlegungen dargestellt, was aus den überlebenden Hunden von der Südpolreise und den verbliebenden Hunden in Framheim künftig geschehen soll. Durch Zufall ergibt es sich, dass der Australier Douglas Mawson Amundsens Hunde gerne für seine eigene Antarktisexpedition (1911-1914) übernehmen möchte. Wegen Krankheiten gibt es auf der Rückreise etliche Verluste unter den Hunden, so dass nach Abgabe von 21 Hunden an Mawson am Ende schließlich nur drei Hunde in Norwegen ankommen, die bei Amundsen und schließlich bei dem Expeditionsmitglied Oskar Wisting ihr Gnadenbrot erhalten.

Im letzten Kapitel geht Mary Tahan nochmals zusammenfassend auf Amundsens Methode seiner Hundeschlittenreisen ein: Zucht von gutem Nachwuchs, liebevolle Pflege und Beschreibung der Hunde als treue Kameraden des Menschen, schließlich der Umschlag von Amundsens Haltung gegenüber den Hunden, als es zum Südpol geht. Nun zählen die Hunde nur noch als Bestandteil der Ausrüstung, der man sich entledigt, wenn sie nicht mehr zu gebrauchen ist. Auch stellt sich die Autorin die kritische Frage, warum Amundsen im „Butcher's Shop“ so viele Hunde hat töten lassen, denn nicht alle wurden als Futter gebraucht! Als erster am Südpol zu sein, war sein oberstes Ziel, koste es was es wolle, in dem Fall die Hunde! Ihnen hat die Autorin in diesem Buch wahrlich ein würdiges Denkmal gesetzt. Das Buch ist jedem zu empfehlen, der sich mit Amundsens Methode der Schlittenreisen näher auseinandersetzen möchte, so traurig der Inhalt auch sein mag.

Begutachtung. This paper was edited by Bernhard Diekmann. 